\title{
Effect of salt substitution on community-wide blood pressure and hypertension incidence
}

\author{
Antonio Bernabe-Ortiz', Víctor G. Sal y Rosas², Vilarmina Ponce-Lucero ${ }^{1}{ }^{1}$, María K. Cárdenas', \\ Rodrigo M. Carrillo-Larco 1,3, Francisco Diez-Canseco1, M. Amalia Pesantes', Katherine A. Sacksteder ${ }^{4}$, \\ Robert H. Gilman ${ }^{4}$ and J. Jaime Miranda ${ }^{1,5 \star}{ }^{1,}$
}

\begin{abstract}
Replacement of regular salt with potassium-enriched substitutes reduces blood pressure in controlled situations, mainly among people with hypertension. We report on a population-wide implementation of this strategy in a stepped-wedge cluster randomized trial (NCT01960972). The regular salt in enrolled households was retrieved and replaced, free of charge, with a combination of $75 \% \mathrm{NaCl}$ and $25 \% \mathrm{KCl}$. A total of 2,376 participants were enrolled in 6 villages in Tumbes, Peru. The fully adjusted intention-to-treat analysis showed an average reduction of $1.29 \mathrm{~mm} \mathrm{Hg}(95 \%$ confidence interval $(95 \% \mathrm{Cl})(-2.17,-0.41))$ in systolic and $0.76 \mathrm{~mm} \mathrm{Hg}(95 \% \mathrm{Cl}(-1.39,-0.13))$ in diastolic blood pressure. Among participants without hypertension at baseline, in the time- and cluster-adjusted model, the use of the salt substitute was associated with a $51 \%(95 \% \mathrm{Cl}$ (29\%, $66 \%)$ ) reduced risk of developing hypertension compared with the control group. In 24-h urine samples, there was no evidence of differences in sodium levels (mean difference $0.01 ; 95 \% \mathrm{Cl}(0.25,-0.23)$ ), but potassium levels were higher at the end of the study than at baseline (mean difference $0.63 ; 95 \% \mathrm{Cl}(0.78,0.47)$ ). Our results support a case for implementing a pragmatic, population-wide, salt-substitution strategy for reducing blood pressure and hypertension incidence.
\end{abstract}

A reduction in salt intake has been identified as one of the most cost-effective measures for improving health outcomes ${ }^{1-3}$. Different studies have reported the benefit of salt-reduction interventions in decreasing blood pressure and cardiovascular events $^{4-6}$. Results from a meta-analysis show that modest reductions in salt intake are followed by a decrease in blood pressure levels among both hypertensive and normotensive subjects ${ }^{7}$. Nevertheless, the evidence of the effectiveness of population-level, behavior change interventions on reducing salt intake is inconsistent, suggesting that education and awareness-raising interventions alone are not sufficient for reducing population salt intake . $^{8}$

Salt substitutes, that is, salt enriched with potassium or other similar components such as magnesium or aluminum, have been reported to be effective in reducing both systolic blood pressure (SBP) and diastolic blood pressure (DBP) ${ }^{9-11}$. Under controlled conditions, salt-substitution strategies can reduce the SBP up to $5 \mathrm{~mm} \mathrm{Hg}$ and the DBP up to $1.5 \mathrm{~mm} \mathrm{Hg}$, and this effect was larger among individuals with hypertension than among normotensive subjects $^{12}$. There is limited evidence, however, from studying the population-level effect of these salt-substitution interventions. A cluster randomized trial conducted in China, evaluating the effect of a community-based sodium reduction program using a salt substitute on salt consumption and blood pressure, found reductions in urinary sodium excretion but not in blood pressure ${ }^{13}$.

Currently, an increasing number of countries have adopted national salt-reduction strategies ${ }^{14}$. Salt-substitution initiatives could aid such strategies in settings where added salt during cooking is the main source of salt intake, particularly in low- and middle-income countries where hypertension rates are increasing at a fast rate ${ }^{15}$. The aim of the present study was to assess the efficacy of a pragmatic intervention using a salt-substitution strategy to reduce blood pressure, as well as its impact on the incidence of hypertension, at the population level, using a stepped-wedge cluster trial in Peru.

\section{Results}

Population characteristics. Figure 1 shows the details of participants' enrollment, including dates, number of subjects assessed, those lost to follow-up and those analyzed for each step of the trial. A total of 2,376 (91.2\%) out of 2,605 eligible subjects in the 6 villages were enrolled in the study from 2 April to 17 July, 2014: 49.6\% females, mean age $43.3 \pm 17.2$ years.

Of note, only $18.9 \%$ of the individuals had $\geq 12$ years of education, $68.1 \%$ were in the overweight or obesity range with a body mass index (BMI) $\geq 25 \mathrm{~kg} \mathrm{~m}^{-2}$ and $18.3 \%$ had a diagnosis of hypertension. Table 1 shows the characteristics of the study population at baseline and a comparison between the control and the intervention periods. There were differences among villages in the distribution of age, education, wealth index, BMI, SBP, DBP and hypertension (see Supplementary Table 1).

Effect of the salt substitute on blood pressure levels. In the intentto-treat analysis, adjusting only for clustering and time effects, there was an average reduction of $1.23 \mathrm{~mm} \mathrm{Hg}(95 \% \mathrm{CI}(0.38,2.07)$; $P=0.004)$ in SBP and $0.72 \mathrm{~mm} \mathrm{Hg}(95 \% \mathrm{CI}(0.10,1.34) ; P=0.022)$ in DBP among the participants who received the salt substitute compared with controls. These results remained consistent after further adjustment for sex, age, years of education, wealth index and BMI measured at baseline (Table 2).

Variations in SBP and DBP mean levels over the intervention and control periods are shown in Fig. 2. There was no evidence that 


\begin{tabular}{|c|c|c|c|c|c|c|}
\hline $\begin{array}{c}\text { Step } 0 \\
2 \text { April } 2014 \text { to } \\
17 \text { July } 2014\end{array}$ & $\begin{array}{c}\text { Step 1 } \\
8 \text { August } 2014 \text { to } \\
11 \text { February } 2015\end{array}$ & $\begin{array}{c}\text { Step } 2 \\
12 \text { February } 2015 \text { to } \\
13 \text { July } 2015\end{array}$ & $\begin{array}{c}\text { Step } 3 \\
14 \text { July } 2015 \text { to } \\
18 \text { January } 2016\end{array}$ & $\begin{array}{c}\text { Step } 4 \\
19 \text { January } 2016 \text { to } \\
21 \text { May } 2016\end{array}$ & $\begin{array}{c}\text { Step } 5 \\
22 \text { May } 2016 \text { to } \\
21 \text { October } 2016\end{array}$ & $\begin{array}{c}\text { Step } 6 \\
22 \text { October } 2016 \text { to } \\
14 \text { March } 2017\end{array}$ \\
\hline \multirow[t]{6}{*}{$\begin{aligned} 6 & \text { villages } \\
2,376 & \text { subjects }\end{aligned}$} & \multirow[t]{5}{*}{$\begin{aligned} 5 & \text { villages } \\
1,840 & \text { subjects } \\
183 & \text { LTFU } \\
1,657 & \text { analyzed }\end{aligned}$} & \multirow[t]{3}{*}{$\begin{aligned} 4 & \text { villages } \\
1,393 & \text { subjects } \\
146 & \text { LTFU } \\
1,247 & \text { analyzed }\end{aligned}$} & \multirow[t]{2}{*}{$\begin{aligned} 3 & \text { villages } \\
1,064 & \text { subjects } \\
125 & \text { LTFU } \\
939 & \text { analyzed }\end{aligned}$} & $\begin{aligned} 2 & \text { villages } \\
650 & \text { subjects } \\
52 & \text { LTFU } \\
598 & \text { analyzed }\end{aligned}$ & $\begin{aligned} 1 & \text { villages } \\
322 & \text { subjects } \\
30 & \text { LTFU } \\
292 & \text { analyzed } \\
& \end{aligned}$ & $\begin{aligned} 322 & \text { subjects } \\
35 & \text { LTFU } \\
237 & \text { analyzed }\end{aligned}$ \\
\hline & & & & Cohort 4: 1 village & $\begin{aligned} 328 & \text { subjects } \\
29 & \text { LTFU } \\
299 & \text { analyzed }\end{aligned}$ & $\begin{aligned} 328 & \text { subjects } \\
28 & \text { LTFU } \\
300 & \text { analyzed }\end{aligned}$ \\
\hline & & & Cohort 3: 1 village & $\begin{aligned} 414 & \text { subjects } \\
61 & \text { LTFU } \\
353 & \text { analyzed }\end{aligned}$ & $\begin{aligned} 414 & \text { subjects } \\
68 & \text { LTFU } \\
346 & \text { analyzed }\end{aligned}$ & $\begin{aligned} 414 & \text { subjects } \\
84 & \text { LTFU } \\
330 & \text { analyzed }\end{aligned}$ \\
\hline & & Cohort 2: 1 village & $\begin{aligned} 329 & \text { subjects } \\
8 & \text { LTFU } \\
301 & \text { analyzed }\end{aligned}$ & $\begin{aligned} 329 & \text { subjects } \\
27 & \text { LTFU } \\
302 & \text { analyzed }\end{aligned}$ & $\begin{aligned} 329 & \text { subjects } \\
27 & \text { LTFU } \\
302 & \text { analyzed }\end{aligned}$ & $\begin{aligned} 329 & \text { subjects } \\
29 & \text { LTFU } \\
300 & \text { analyzed }\end{aligned}$ \\
\hline & & $\begin{aligned} 447 & \text { subjects } \\
30 & \text { LTFU } \\
417 & \text { analyzed }\end{aligned}$ & $\begin{aligned} 447 & \text { subjects } \\
51 & \text { LTFU } \\
396 & \text { analyzed }\end{aligned}$ & $\begin{aligned} 447 & \text { subjects } \\
34 & \text { LTFU } \\
413 & \text { analyzed }\end{aligned}$ & $\begin{aligned} 447 & \text { subjects } \\
43 & \text { LTFU } \\
404 & \text { analyzed }\end{aligned}$ & $\begin{aligned} 447 & \text { subjects } \\
62 & \text { LTFU } \\
385 & \text { analyzed }\end{aligned}$ \\
\hline & $\begin{aligned} 536 & \text { subjects } \\
54 & \text { LTFU } \\
482 & \text { analyzed }\end{aligned}$ & $\begin{aligned} 536 & \text { subjects } \\
46 & \text { LTFU } \\
490 & \text { analyzed }\end{aligned}$ & $\begin{aligned} 536 & \text { subjects } \\
56 & \text { LTFU } \\
480 & \text { analyzed }\end{aligned}$ & $\begin{aligned} 536 & \text { subjects } \\
44 & \text { LTFU } \\
492 & \text { analyzed }\end{aligned}$ & $\begin{aligned} 536 & \text { subjects } \\
57 & \text { LTFU } \\
479 & \text { analyzed }\end{aligned}$ & $\begin{aligned} 536 & \text { subjects } \\
77 & \text { LTFU } \\
459 & \text { analyzed }\end{aligned}$ \\
\hline
\end{tabular}

Fig. 1 | Flowchart of participants in the stepped-wedge trial. LTFU, lost to follow-up. Randomization of villages occurred after baseline assessment.

the effect of the intervention was modified over time $(P=0.14$ for SBP and 0.46 for DBP). Mean levels of SBP and DBP by village and intervention period are available in the supplementary files (see Extended Data Figs. 1 and 2, respectively).

Results from exploratory analyses, shown in Table 3, showed no evidence of an interaction effect by subgroup. When the analysis was stratified by hypertension status at baseline, an average reduction in SBP of $1.92 \mathrm{~mm} \mathrm{Hg}(95 \%$ CI $(0.54,3.29))$ and $1.18 \mathrm{~mm} \mathrm{Hg}$ $(95 \%$ CI $(0.08,2.29))$ in DBP among individuals with hypertension at baseline was observed. Among individuals without hypertension, the corresponding average reductions in SBP and DBP were $1.15 \mathrm{~mm} \mathrm{Hg}(95 \% \mathrm{CI}(0.34,1.96))$ and $0.63 \mathrm{~mm} \mathrm{Hg}(95 \% \mathrm{CI}$ $(-0.01,1.28))$, respectively. In addition, participants did not report medication changes for the duration of the study $(10.5 \%$ at baseline versus $10.1 \%$ at the end of the study $(P=0.73)$ ). In terms of age subgroups, the effect on blood pressure among those aged $\geq 60$ years was a reduction of $2.17 \mathrm{~mm} \mathrm{Hg}(95 \%$ CI $(0.68,3.67))$ in SBP and $1.18 \mathrm{~mm} \mathrm{Hg}(95 \% \mathrm{CI}(0.22,2.14))$ in DBP (Table 3$)$. As a sensitivity analysis, we evaluated a possible interaction between duration of exposure and intervention effect, and did not find evidence of delayed effects (see Supplementary Table 2).

Effect of salt-substitution intervention on the incidence of hypertension. After excluding patients with hypertension at baseline $(n=428)$, the data for 1,891 of the 1,914 subjects were available for analysis with 4,673.4 person-years of follow-up. The overall incidence of hypertension was estimated as 5.1 per 100 person-years (95\% CI $(4.5,5.8))$. In the time- and cluster-adjusted model, the participants were $51 \%$ less likely (hazard ratio $(\mathrm{HR})=0.49,95 \%$ CI $(0.34,0.71)$, $P<0.001)$ to develop hypertension in the intervention period than in the control period (see Extended Data Fig. 3 and Supplementary Table 3). The Schoenfeld residual test for non-proportional hazards was not significant $(P=0.40)$. The observed effect of the intervention remained consistent after further adjustment for sex, age, education, wealth index and BMI $(\mathrm{HR}=0.45 ; 95 \% \mathrm{CI}(0.31,0.66), P<0.001)$.
Effect of salt-substitution intervention on urinary sodium and potassium. The levels of sodium in 24 -h urine samples (see Supplementary Table 4) at the end of the study and baseline were $3.95 \pm 1.83$ (s.d.) g and $3.94 \pm 1.86 \mathrm{~g}$, respectively, and mean difference 0.01 (95\% CI $(0.25,-0.23))$. These results were similar across all the study villages. In contrast, the levels of potassium were higher at the end of the study $(2.60 \pm 1.20 \mathrm{~g})$ than at baseline $(1.97 \pm 1.20 \mathrm{~g})$, and mean difference $0.63(95 \%$ CI $(0.78,0.47))$.

Harms. No severe adverse effects were reported for the duration of the study.

\section{Discussion}

Evidence has been inconclusive regarding the efficacy of population-wide salt-reduction interventions on blood pressure. In the present study, we report the findings of a pragmatic strategy to reduce blood pressure and hypertension incidence at the population level. Overall, there was a decrease in SBP and DBP after the intervention in the entire population, with results suggesting a larger effect among those individuals with hypertension at baseline and older participants. In addition to the effect on blood pressure levels, the risk of incidence of hypertension was halved among those who received the intervention. These findings are supported by the analysis of urine samples, at baseline and follow-up, which showed an increase in mean potassium levels but no changes in sodium levels, indicating that the salt-substitution intervention was accepted and adopted. We did not find evidence of a delayed effect, suggesting that the effect is sustainable, independently of the duration of the intervention, given the nature of the stepped-wedge design.

The absolute reductions in blood pressure may appear modest, yet they carry major implications. The Prospective Studies Collaboration conducted a meta-analysis of 61 observational studies of blood pressure and vascular disease in adults and found that, for each $2 \mathrm{~mm} \mathrm{Hg}$ decrease in SBP, stroke mortality and cardiovascular mortality decreased by $10 \%$ and $7 \%$, respectively, an effect 


Table 1 | Description of the study population at baseline by
control and intervention periods
\begin{tabular}{llll} 
Variables & Baseline & Time in & \\
\cline { 2 - 3 } & $N=2376$ & $\begin{array}{l}\text { Control } \\
\text { (person-years) }\end{array}$ & $\begin{array}{l}\text { Intervention } \\
\text { (person-years) }\end{array}$
\end{tabular}

Sex

$\begin{array}{llll}\text { Female } & 1,197(50.4) & 1,335.2 & 17,68.4 \\ \text { Male } & 1,179(49.6) & 1,212.0 & 18,36.9\end{array}$

Age (years)

Mean (s.d.) $\quad 43.3(17.2)$

18-29 $633(26.6)$

30-44

780 (32.8)

595.6

703.0

45-64

$\geq 65$

656 (27.6)

880.2

$1,226.9$

715.3

$1,129.2$

546.3

Wealth index

\section{Bottom}

689 (29.6)

356.1

$1,137.8$

Middle

785 (33.7)

629.4

$1,180.6$

Top

855 (36.7)

866.5

$1,232.5$

Education (years)

$<7$

7-11

$\geq 12$

$836(35.2) \quad 909.0$

$1,281.0$

$1,636.6$

687.6

Study site (village)

A

B

C

D

E

F

1,090 (45.9) $\quad 1,185.3$

$450(18.9) \quad 452.9$

$1,366.1$

$536(22.6) \quad 1.7$

883.1

447 (18.8)

286.9

518.3

414 (17.4)

329.0

460.2

328 (13.8)

542.1

256.3

$322(13.6)$

750.6

121.3

BMI $\left(\mathrm{kg} \mathrm{m}^{-2}\right)$

Mean (s.d.)

$27.2(4.6)$

Normal weight

$758(32.7)$

Overweight

$985(42.5)$

762.3

$1,160.1$

Obese

$573(24.7)$

$1,093.0$

$1,492.2$

887.0

Blood pressure $(\mathrm{mm} \mathrm{Hg})$

SBP (mean (s.d.)) $113.1(17.0)$

$\mathrm{DBP}$ (mean (s.d.)) $72(10.1)$

Hypertension

$\begin{array}{llll}\text { No } & 1,914(81.7) & 2,038.0 & 2,925.6 \\ \text { Yes } & 428(18.3) & 476.1 & 646.2\end{array}$

that was observed in reductions of SBP levels up to $115 \mathrm{~mm} \mathrm{Hg}^{16}$. This indicates that small reductions in blood pressure at the population level could result in large public health gains, in line with the approaches to shift the entire distribution of a given risk factor $^{17}$. The main challenge until now, however, has been how best to introduce and achieve these changes under real-life conditions. The present study demonstrates that such benefits can be introduced at a population-wide level.

Salt substitutes have been previously tested, mostly in China and mainly on patients with established hypertension ${ }^{12}$, and they show reductions in blood pressure, with a larger effect observed among individuals with hypertension. Similar results have been obtained using home blood pressure measurements ${ }^{18}$. The present study
Table 2 | Overall effect of the intervention on blood pressure levels

\begin{tabular}{|c|c|c|c|c|}
\hline \multirow{2}{*}{$\begin{array}{l}\text { Blood } \\
\text { pressure } \\
\text { levels }\end{array}$} & \multicolumn{2}{|c|}{$\begin{array}{l}\text { Time- and cluster-adjusted } \\
\text { estimates }^{\mathrm{a}}\end{array}$} & \multicolumn{2}{|c|}{ Fully adjusted estimates ${ }^{b}$} \\
\hline & Coefficient $(95 \% \mathrm{Cl})$ & $P$ & Coefficient $(95 \% \mathrm{CI})$ & $P$ \\
\hline \multicolumn{5}{|c|}{ Main analysis } \\
\hline SBP & $-1.23(-2.07,-0.38)$ & 0.004 & $-1.29(-2.17,-0.41)$ & 0.004 \\
\hline DBP & $-0.72(-1.34,-0.10)$ & 0.022 & $-0.76(-1.39,-0.13)$ & 0.017 \\
\hline
\end{tabular}

A linear mixed effects regression model was used for analyses $(n=2,376$ biologically independent individuals and 16,632 samples in total). ${ }^{\mathrm{a} A d j u s t e d ~ f o r ~ t i m e ~ a n d ~ c l u s t e r i n g, ~ p e r ~ s t u d y ~ d e s i g n . ~}$ ${ }^{\mathrm{b}}$ Adjusted for time and clustering, but also for age, sex, education, wealth index and BMI.

further expands the current literature by using a pragmatic population-wide intervention that included a heterogeneous sample; that is, the intervention was delivered to the general population irrespective of hypertension status (participants were or were not aware of the diagnosis), and perhaps, due to this, the effect was modest (that is, a differential effect of salt substitute in subjects with hypertension diagnosis compared with those with a recent diagnosis and those without the diagnosis). These features account for the potential scalability of our results to large populations and their influence on public health policies. The present study introduced a salt substitute containing $\mathrm{NaCl}(75 \%)$ and $\mathrm{KCl}(25 \%)^{19}$; however, previous reports have also included other minerals (for example, $\left.\mathrm{MgSO}_{4}\right)^{12,18}$. Potassium has also been shown to have benefits for blood pressure, irrespective of the lowering of sodium ${ }^{20-22}$, especially among individuals with hypertension and a high consumption of sodium ${ }^{23}$. Hence, the potassium contained in the salt substitute could contribute to the explanation of the benefits observed in the present study. This hypothesis is further supported by the higher levels of potassium, but not sodium, excretion in the urine samples at the end of the study. A higher intake of potassium could be achieved through a combined strategy of a salt-substitution intervention and health education programs that focus on promoting the consumption of fresh vegetables and fruit to increase potassium intake.

Our results also point to a lower incidence of hypertension in the participants receiving the intervention, a key clinical and public health finding. Whether this is a short-term effect (that is, the intervention did not prevent hypertension onset but rather delayed it) remains to be studied further. Because the endocrine system in charge of salt regulation, the renin-angiotensin-aldosterone system, continues to receive larger amounts of sodium or lower amounts of potassium, it is probable that blood pressure will start to increase until it reaches hypertensive thresholds ${ }^{24}$. Longer follow-ups, with and without intervention, are required to assess whether the endocrine system develops salt resistance. However, our findings show no evidence of an interaction between time and intervention.

From the viewpoint of pragmatic implementation, we provide evidence for the ability to introduce a salt substitute to entire participating communities after a social marketing campaign designed to improve its acceptance. With evidence that antihypertensive medication is often unavailable or unaffordable in many low- and middle-income settings ${ }^{25}$, the implementation of a similar primary prevention strategy could reduce the burden associated with hypertension and its cardiovascular complications. The cost of the salt substitute should also be considered. During the present study, before the intervention the cost of $1 \mathrm{~kg}$ of the salt substitute to the general public was $35 \mathrm{PEN}(\sim \$ \mathrm{US} 10)$, and through the project we were able to achieve a price reduction to $14 \mathrm{PEN}(\sim \$ \mathrm{US} 4)$, further indicating opportunities for scaling up implementation efforts.

Current hypertension guidelines advocate non-pharmacologic treatment, even in patients with low-risk, stage 1 hypertension ${ }^{26}$. 



Fig. 2 | Trends in mean SBP and DBP. a,b, Trends in mean SBP (a) and DBP (b) and their respective 95\% Cls by intervention or control group. Time periods are 5-month analysis periods occurring before the initiation of the intervention in each wave $(n=2,072)$.

Our results provide evidence of a pragmatic approach that reduces blood pressure and, secondarily, halves the incidence of hypertension. These guidelines have also lowered the threshold for hypertension, meaning that more people will receive this diagnosis and need to incorporate essential hypertension management strategies, making it difficult for health systems to provide pharmacologic treatment and counseling to new patients. Moreover, given the alarming rates of non-adherence to medication for hypertension globally, non-pharmacologic measures to improve blood pressure control at the population level are urgently needed. This population-wide intervention has the potential to contribute to reducing overall blood pressure levels without additional overcrowding of the healthcare system, potentially saving healthcare costs ${ }^{27}$.

In Peru, and in many other resource-constrained settings, there are different venues through which a salt substitute can be introduced to replace the current salt, including, for example, community kitchens for people of low socioeconomic status ${ }^{28}$, a different national program for providing nutritious breakfasts for children attending public schools in rural areas or elderly people. Therefore, the logistics underlying this process are already in place and could be adapted to provide patients with hypertension, and their families, with a salt substitute. Similar scenarios may be present in other countries, signaling a window of opportunity to introduce a seemingly effective tool to reduce blood pressure. Other countries, including high-income countries, could also accommodate a similar salt-substitution approach using existing channels and various venues, including school feeding programs.

The present study is an intervention study that provides a high level of evidence. The randomized allocation of the intervention removes several biases that exist in non-randomized studies, even after adjusting for potential confounders. The stepped-wedge design guarantees a pragmatic scheme in which randomization of the intervention is protected, allowing a large population to be reached. In some villages a reduction in blood pressure was observed before the intervention, and potential explanations for this could be a community-like white coat effect, which, in the case of rural or semi-urban areas with limited access to healthcare, can also be present. Another explanation could be regression to the mean. In both circumstances, the repeated measurements would be the best way to overcome such potential weaknesses. The repeated follow-up visits over short, equally spaced periods, a characteristic of the stepped-wedge design, augmented the statistical power and afforded additional strength to address regression to the mean. In addition, uptake of the intervention was objectively assessed, with urine samples demonstrating more potassium excretion at the end of the intervention, suggesting that the intervention was indeed well received and the salt substitute used. Consistently, the intervention included a social marketing campaign to guarantee adoption of the new salt. From a public health perspective, the delivery of the intervention at the population level following a pragmatic methodology could inform prevention guidelines and policies to control the rising burden of increased blood pressure worldwide. Nevertheless, the limitations of the study must also be acknowledged. First, the absence of a dietary assessment of other sources of sodium and potassium could have an impact on our results; however, this factor should be negligible because of the population-wide approach used. We provided whole villages with the salt substitute, also targeting families who prepare and sell food as street vendors. Therefore, it seems unlikely that other sources of sodium could have contaminated the intervention. Similarly, it is also unlikely that other sources of potassium confounded the intervention. To prevent any potential harm, and thus protect the safety of the study population, we did not include people with kidney disease or those receiving digoxin (used as a proxy of cardiovascular disease). Although this exclusion warrants close follow-up of these patients, including regular check-ups with their physicians or tailored diets, it does not affect the implementation of wider population-wide benefits aimed at lowering blood pressure. Finally, despite the inclusion of urine data from individuals with a complete 24-h urine sample, the levels of creatinine appear to be somewhat lower at baseline than during follow-up, thus reflecting some under-collection at the beginning of the study. However, we believe that this may have a negligible effect, because the creatinine levels were in the normal range. Moreover, the variation observed in the levels of creatinine was within the range of dispersion (SD) of measurements.

Our results provide evidence that a population-based intervention to replace regular salt with a low-sodium, potassiumenriched salt reduces both SBP and DBP, particularly in people with hypertension. In addition, the intervention halved the incidence of 


\begin{tabular}{|c|c|c|c|c|}
\hline \multirow{2}{*}{$\begin{array}{l}\text { Blood } \\
\text { pressure } \\
\text { levels }\end{array}$} & \multicolumn{2}{|c|}{$\begin{array}{l}\text { Time- and cluster-adjusted } \\
\text { estimates }^{\mathrm{a}}\end{array}$} & \multicolumn{2}{|c|}{ Fully adjusted estimates $^{b}$} \\
\hline & Coefficient $(95 \% \mathrm{Cl})$ & $P$ & Coefficient $(95 \% \mathrm{Cl})$ & $P$ \\
\hline \multicolumn{5}{|c|}{ Hypertension ${ }^{c}$} \\
\hline \multicolumn{5}{|c|}{ Among individuals without hypertension } \\
\hline SBP & $-1.13(-1.93,-0.33)$ & 0.006 & $-1.15(-1.96,-0.34)$ & 0.005 \\
\hline DBP & $-0.62(-1.23,0)$ & 0.051 & $-0.63(-1.28,0.01)$ & 0.053 \\
\hline \multicolumn{5}{|c|}{ Among individuals with hypertension } \\
\hline SBP & $-1.74(-3.04,-0.44)$ & 0.009 & $\begin{array}{l}-1.92(-3.29 \\
-0.54)\end{array}$ & 0.006 \\
\hline DBP & $-1.25(-2.24,-0.27)$ & 0.013 & $-1.18(-2.29,-0.08)$ & 0.036 \\
\hline \multicolumn{5}{|l|}{$\operatorname{Age}^{d}$} \\
\hline \multicolumn{5}{|c|}{ Among individuals aged $<40$ years } \\
\hline SBP & $-0.91(-1.51,-0.31)$ & 0.003 & $\begin{array}{l}-0.94(-1.54 \\
-0.34)\end{array}$ & 0.002 \\
\hline DBP & $-0.25(-0.79,0.30)$ & 0.38 & $-0.27(-0.80,0.27)$ & 0.33 \\
\hline \multicolumn{5}{|c|}{ Among individuals aged $40-59$ years } \\
\hline SBP & $-1.20(-2.02,-0.38)$ & 0.004 & $-1.17(-1.98,-0.35)$ & 0.005 \\
\hline DBP & $-1.04(-1.70,-0.39)$ & 0.002 & $-1.01(-1.67,-0.36)$ & 0.002 \\
\hline \multicolumn{5}{|c|}{ Among individuals aged $\geq 60$ years } \\
\hline SBP & $-1.95(-3.44,-0.45)$ & 0.01 & $-2.17(-3.67,-0.68)$ & 0.004 \\
\hline DBP & $-1.13(-2.09,-0.18)$ & 0.02 & $-1.18(-2.14,-0.22)$ & 0.02 \\
\hline
\end{tabular}

A linear mixed effects regression model was used for analyses $(n=2,376$ biologically independent individuals and 16,632 samples in total). ${ }^{\mathrm{a}}$ Adjusted for time and clustering, per study design. ${ }^{b}$ Adjusted for time and clustering, but also for age, sex, education, wealth index and BMI. Age was excluded as a confounder when analyses were stratified by age. ${ }^{c} P$ values for the interaction of hypertension status and intervention were 0.858 and 0.951 for the SBP and DBP models. ${ }^{d} P$ values for the interaction of age groups and intervention were 0.211 and 0.279 , and 0.379 and 0.015 for the SBP and DBP models.

hypertension. This pragmatic intervention could be adapted and scaled up to counter the high burden of elevated blood pressure currently observed worldwide.

\section{Online content}

Any methods, additional references, Nature Research reporting summaries, source data, extended data, supplementary information, acknowledgements, peer review information; details of author contributions and competing interests; and statements of data and code availability are available at https://doi.org/10.1038/s41591020-0754-2.

Received: 6 August 2019; Accepted: 7 January 2020; Published online: 17 February 2020

\section{References}

1. Wang, G. \& Bowman, B. A. Recent economic evaluations of interventions to prevent cardiovascular disease by reducing sodium intake. Curr. Atheroscler. Rep. 15, 349 (2013).

2. Salt Reduction: Fact Sheet (World Health Organization, 2016); https://www. who.int/news-room/fact-sheets/detail/salt-reduction

3. Kontis V. et al. Three public health interventions could save 94 million lives in 25 years global impact assessment analysis. Circulation 140, 715-725 (2019).

4. Melander, O. et al. Moderate salt restriction effectively lowers blood pressure and degree of salt sensitivity is related to baseline concentration of renin and $\mathrm{N}$-terminal atrial natriuretic peptide in plasma. J. Hypertens. 25, 619-627 (2007).
5. Murray, C. J. et al. Effectiveness and costs of interventions to lower systolic blood pressure and cholesterol: a global and regional analysis on reduction of cardiovascular-disease risk. Lancet 361, 717-725 (2003).

6. Sacks, F. M. et al. Effects on blood pressure of reduced dietary sodium and the dietary approaches to stop hypertension (DASH) diet. DASH-Sodium Collaborative Research Group. N. Engl. J. Med. 344, 3-10 (2001).

7. He, F. J., Li, J. \& Macgregor, G. A. Effect of longer term modest salt reduction on blood pressure: Cochrane systematic review and meta-analysis of randomised trials. BMJ 346, f1325 (2013).

8. Trieu, K. et al. Review of behaviour change interventions to reduce population salt intake. Int. J. Behav. Nutr. Phys. Act. 14, 17 (2017).

9. China Salt Substitute Study Collaborative Group. Salt substitution: a low-cost strategy for blood pressure control among rural Chinese. A randomized, controlled trial. J. Hypertens. 25, 2011-2018 (2007).

10. Geleijnse, J. M., Witteman, J. C., Bak, A. A., den Breeijen, J. H. \& Grobbee, D. E. Reduction in blood pressure with a low sodium, high potassium, high magnesium salt in older subjects with mild to moderate hypertension. BMJ 309, 436-440 (1994).

11. Zhou, B. et al. Long-term effects of salt substitution on blood pressure in a rural north Chinese population. J. Hum. Hypertens. 27, 427-433 (2013).

12. Peng, Y. G. et al. Effects of salt substitutes on blood pressure: a metaanalysis of randomized controlled trials. Am. J. Clin. Nutr. 100, 1448-1454 (2014).

13. Li, N. et al. The effects of a community-based sodium reduction program in rural China-a cluster-randomized trial. PLoS ONE 11, e0166620 (2016).

14. Trieu, K. et al. Salt reduction initiatives around the world-a systematic review of progress towards the global target. PLOS ONE 10, e0130247 (2015).

15. NCD Risk Factor Collaboration. Worldwide trends in blood pressure from 1975 to 2015: a pooled analysis of 1479 population-based measurement studies with 19.1 million participants. Lancet 389, 37-55 (2017).

16. Lewington, S. et al. Age-specific relevance of usual blood pressure to vascular mortality: a meta-analysis of individual data for one million adults in 61 prospective studies. Lancet 360, 1903-1913 (2002).

17. Rose, G. Sick individuals and sick populations. Int. J. Epidemiol. 14, 32-38 (1985).

18. Hu, J., Zhao, L., Thompson, B., Zhang, Y. \& Wu, Y. Effects of salt substitute on home blood pressure differs according to age and degree of blood pressure in hypertensive patients and their families. Clin. Exp. Hypertens. 40, 664-672 (2018)

19. Saavedra-Garcia, L. et al. Applying the triangle taste test to assess differences between low sodium salts and common salt: evidence from Peru. PLoS ONE 10, e0134700 (2015).

20. Binia, A., Jaeger, J., Hu, Y., Singh, A. \& Zimmermann, D. Daily potassium intake and sodium-to-potassium ratio in the reduction of blood pressure: a meta-analysis of randomized controlled trials. J. Hypertens. 33, 1509-1520 (2015).

21. Mente, A. et al. Urinary sodium excretion, blood pressure, cardiovascular disease, and mortality: a community-level prospective epidemiological cohort study. Lancet 392, 496-506 (2018).

22. Poorolajal, J. et al. Oral potassium supplementation for management of essential hypertension: a meta-analysis of randomized controlled trials. PLoS ONE 12, e0174967 (2017).

23. Filippini, T., Violi, F., D’Amico, R. \& Vinceti, M. The effect of potassium supplementation on blood pressure in hypertensive subjects: a systematic review and meta-analysis. Int. I. Cardiol. 230, 127-135 (2017).

24. Perez, V. \& Chang, E. T. Sodium-to-potassium ratio and blood pressure, hypertension, and related factors. Adv. Nutr. 5, 712-741 (2014).

25. Attaei, M. W. et al. Availability and affordability of blood pressure-lowering medicines and the effect on blood pressure control in high-income, middle-income, and low-income countries: an analysis of the PURE study data. Lancet Public Health 2, e411-e419 (2017).

26. Whelton, P. K. et al. 2017 ACC/AHA/AAPA/ABC/ACPM/AGS/APhA/ASH/ ASPC/NMA/PCNA guideline for the prevention, detection, evaluation, and management of high blood pressure in adults: executive summary: a report of the American College of Cardiology/American Heart Association Task Force on Clinical Practice Guidelines. J. Am. Coll. Cardiol. 71, 2199-2269 (2018).

27. Aminde, L. N., Takah, N. F., Zapata-Diomedi, B. \& Veerman, J. L. Primary and secondary prevention interventions for cardiovascular disease in low-income and middle-income countries: a systematic review of economic evaluations. Cost Eff. Resour. Alloc. 16, 22 (2018).

28. Garret J. L. Comedores Populares: Lessons For Urban Programming From Peruvian Community Kitchens (CARE-USA, 2001).

Publisher's note Springer Nature remains neutral with regard to jurisdictional claims in published maps and institutional affiliations.

(c) The Author(s), under exclusive licence to Springer Nature America, Inc. 2020 


\section{Methods}

The study protocol and methods have been described previously ${ }^{29}$, and a summary is provided below. The CONSORT statement for randomized cluster trials ${ }^{30}$ and recent literature on reporting results of stepped-wedge cluster trials ${ }^{31,32}$ were utilized.

Study design. A stepped-wedge, cluster, randomized controlled trial was conducted, in which the six participating villages (clusters) crossed over from the control to the intervention phase during the study ${ }^{33}$. The order of switchover for each cluster was determined by randomization, and all villages received the salt substitute by the end of the study. The structure of the stepped wedge is provided in Fig. 3, where the intervention periods (village implementation phases, shown in gray) lasted 4 months, and blood pressure measurements were made every 5 months after the baseline period. The study was undertaken between April 2014 (start of baseline assessment) and March 2017 (last measurement and assessment).

Study setting. Tumbes, a coastal region in northern Peru, bordering Ecuador, was the setting selected for the present study because hypertension prevalence and incidence rates are above the national average ${ }^{34,35}$. According to official estimates of the Tumbes population ${ }^{36}$, in 2017 there were 243,362 inhabitants with a life expectancy of 75 years; $20 \%$ of the population did not have any health insurance and $12 \%$ were below the poverty line. The semi-urban area of the region, with approximately 100 villages of varying sizes and approximately 80,000 inhabitants, was the area chosen for the study. Mid-sized villages with 350-700 individuals ( 130-250 households) were initially selected for the study. Of the 20 villages available with these characteristics, 6 were randomly selected. Sufficient distance between them was also ensured (that is, a median of $14 \mathrm{~km}$ (interquartile range 7.1-17.1) between them) to avoid contamination by verifying the selection of villages on the map.

Participants and recruitment. Potentially eligible subjects were identified from the most updated census in the area (2010, updated in 2014). All men and women aged $\geq 18$ years from the six selected villages, who were capable of understanding procedures and of providing informed consent, and full-time residents in the area, were eligible. Individuals with a self-reported history of chronic kidney disease or of heart disease treated with digoxin were excluded from the study.

Participant recruitment, as well as the initial assessment, was performed during the first 4 months of the study (April to July 2014). Individuals were contacted through home visits aiming to enroll all members of households in the villages who met the selection criteria.

Randomization and blinding. The selected villages were randomly assigned to one of the six sequences (one village $=$ one cluster) for time crossover from control to intervention. For this, a computer-generated list of random numbers was used and information was kept in a password-protected computer. The order of the villages to be implemented was revealed one by one as required, according to the nature of the study. Due to the pragmatic nature of the intervention, the participants were not blinded; however, the primary study outcome was objectively measured using standardized techniques. A team of fieldworkers, not involved with the implementation of the intervention, was responsible for periodic assessments of participants using automated devices to reduce observer bias.

Intervention. Through the application of social marketing strategies ${ }^{37}$, a campaign was developed to target women responsible for food preparation at home. The purpose of the marketing campaign was to introduce the salt substitute as a new product in the intervention villages, and enhance its acceptance. Thus, common salt
$(\mathrm{NaCl})$ used in the enrolled households was retrieved and replaced, free of charge, with a salt substitute, using a combination of $75 \% \mathrm{NaCl}$ and $25 \% \mathrm{KCl}$, based on previous research ${ }^{19}$. Iodine, in addition to fluorine, was also part of the salt substitute following Peruvian regulation $\mathrm{s}^{38}$. As the usual cost of a bag of $1 \mathrm{~kg}$ of common salt in the region was between $\$ U S 0.15$ and $\$$ US0.17 (about 0.50 PEN), we provided the salt substitute free of charge to the participants in their respective homes.

The time for provision of a salt replacement was planned to happen over a period of 5 months in each village; however, there was a delay in salt-substitute delivery of, on average, $15 \mathrm{~d}$. The intervention considered making the salt delivery to families, as well as to owners of small shops, bakeries and community kitchens ${ }^{28}$, and food vendors including street vendors and restaurants. This approach was used to guarantee full replacement of salt in the entire village. Additional saltsubstitute packs were also made freely available during the study period in case any household required additional salt.

Outcomes and data collection. The primary outcomes were SBP and DBP, assessed as continuous variables (in $\mathrm{mm} \mathrm{Hg}$ ) evaluated in the period between the end of each wedge and the start of the next one. Blood pressure assessments were performed with the participants seated, after a 5-min resting period, using an automated device (OMRON HEM-780) that had been previously validated in adult populations $^{39}$. Three different measurements, at least $1 \mathrm{~min}$ apart, were carried out, and the average of the second and third measurements was used for the analyses.

The secondary outcomes included progression toward hypertension (incidence) and, in a random subsample of participants, changes in levels of sodium and potassium excretion in the 24-h urine. Hypertension at baseline was defined as $\mathrm{SBP} \geq 140 \mathrm{~mm} \mathrm{Hg}, \mathrm{DBP} \geq 90 \mathrm{~mm} \mathrm{Hg}$, a self-reported physician diagnosis or current treatment for hypertension ${ }^{40}$. During follow-up, hypertension was defined in two ways: considering only the study measurements (average of the second and third measurements, with the participant in seated position, after resting $5 \mathrm{~min}$, and at least $1 \mathrm{~min}$ between measurements) or taking advantage of the repeated assessments conducted every 5 months, as well as using the same definition as in the baseline.

After providing consent, each participant was given a unique code. At baseline, detailed information about sociodemographics (for example, age, sex, education and wealth index), lifestyle behaviors (smoking, alcohol consumption and physical activity), self-reported personal medical history and medication (hypertension and type 2 diabetes mellitus), anthropometric measurements (height, weight and blood pressure), and healthcare utilization and expenditure was collected using paper-based formats. Follow-up assessments were conducted in all participants and included some lifestyle behaviors (smoking and alcohol consumption), anthropometric measurements (weight and blood pressure), and healthcare utilization and expenditure.

Urine samples were retrieved in a random subsample of 600 participants after baseline and in another randomly selected subsample of 600 participants at the end of the study. Only one participant per household was included in the urine assessments. Urine samples were collected over a 24 -h period, and all samples were assessed in a central laboratory facility. These samples were used to extract information about levels of creatinine, sodium and potassium. Sodium and potassium were assessed using the ion-selective electrode method, whereas creatinine was assessed using the compensated kinetic Jaffe method.

Statistical methods. All statistical procedures were conducted using Stata for Windows v.15.0 (StataCorp) and R statistical software ${ }^{41}$, and a per-protocol, intent-to-treat analysis was performed. A pre-specified linear mixed effects regression analysis was performed to model SBP and DBP using an identity link,

\begin{tabular}{|c|c|c|c|c|c|c|c|c|c|c|c|c|c|c|c|c|c|c|c|c|c|c|c|c|}
\hline & \multicolumn{5}{|c|}{2014} & \multicolumn{9}{|c|}{2015} & \multicolumn{8}{|c|}{2016} & \multicolumn{2}{|c|}{2017} \\
\hline Village & 4 & \begin{tabular}{l|l}
5 & \\
\end{tabular} & \begin{tabular}{l|l}
6 & 7 \\
\end{tabular} & 8 & \begin{tabular}{|l|l|l|l|}
9 & 10 & 11 & 12 \\
\end{tabular} & 1 & 2 & 3 & $4: 5$ & 5 & 6 & \begin{tabular}{|l|}
8 \\
\end{tabular} & \begin{tabular}{|l|l|l|}
9 & 10 & 11 \\
\end{tabular} & \begin{tabular}{l|l}
11 & 12 \\
\end{tabular} & 1 & \begin{tabular}{l|l}
2 & 3 \\
\end{tabular} & 4 & 5 & \begin{tabular}{|l|l}
6 & 7 \\
\end{tabular} & 8 & 9 & \begin{tabular}{|l|l|l|}
10 & 11 & 12 \\
\end{tabular} & 1 & \begin{tabular}{l|l}
2 & 3 \\
\end{tabular} \\
\hline A & \multirow{6}{*}{\multicolumn{3}{|c|}{ Baseline }} & & & & & & & & & & & & & & & & & & & & & \\
\hline B & & & & & & & & & & & & & & & & & & & & & & & & \\
\hline $\mathrm{c}$ & & & & & & & & & & & & & & & & & & & & & & & & \\
\hline D & & & & & & $A$ & & & & & A & & & & & & & & & & A & & & A \\
\hline E & & & & & & & & & & & & & & & & & & & & & & & & \\
\hline $\mathrm{F}$ & & & & & & & & & & & & & & & & & & & & & & & & \\
\hline
\end{tabular}

Assessment of participants between implementation in each village Period of intervention

Fig. 3 | Structure and time framework of the stepped-wedge, cluster, randomized controlled trial. The assessment of participants included a short questionnaire, and weight and blood pressure measurements. 
an unstructured working correlation, including covariates for intervention status and time period, which was considered as a factor, and random effects for village family and repeated observations of the same individual over time ${ }^{42,43}$, and robust variances were computed. Thus, the following model was used:

$$
Y_{i j k l}=\mu+\alpha_{i}+\gamma_{j}+\varphi_{k}+\beta_{l}+\theta X_{k l}+\varepsilon_{i j k l}
$$

where $Y_{i j k l}$ is the SBP (or DBP) measured for individual $i$, in family $j$, at cluster $k$ in time $l ; \mu$ is the mean outcome in the control group at baseline; $\alpha_{i}$ is a random intercept of individual $i ; \gamma_{j}$ is a random intercept for family $j ; \varphi_{k}$ is a random intercept for cluster $k ; \beta$ is the effect of time $l ; X_{k l}$ is an indicator of the treatment mode in village $k$ at time $l ; \theta$ is the overall effect of the intervention; and $\varepsilon_{i j k l}$ is the random error for the measurement of individual $i$, in family $j$, at cluster $k$, at time $l$.

We also evaluated, as a sensitivity analysis, whether there was evidence of a delayed effect, that is, an interaction between duration of exposure and intervention $^{42}$, and estimated the effect of the intervention on SBP and DBP, controlling for assumed defined possible confounders: age, sex, education, wealth index and BMI at baseline. Furthermore, we conducted exploratory subgroup analyses by hypertension status and age group defined at baseline.

For incidence calculations, Cox's proportional hazard modeling on a calendar time axis, to account for time trends with random effects that follow gamma distribution for village-level (shared) frailty, was considered to compare the instantaneous risk of hypertension for both the intervention and the control groups $^{44}$. The Schoenfeld residuals were used to test for the non-proportional hazard without considering the frailty term ${ }^{45}$. Time- and cluster-adjusted Cox's models were constructed for the primary analysis, and fully adjusted models were generated to account for confounding variables such as age, sex, education, wealth index and BMI at baseline. Calculations (that is, HRs) were estimated taking into account the clustering of villages; in addition, a time-varying binary covariate tracking intervention status was fit, using definitions of times at risk in each of the periods described above.

Finally, changes in the 24-h urine concentrations of sodium and potassium were also evaluated (at the end of the study and after baseline). For the analysis, we included only individuals with a complete 24 -h urine sample, defined as (1) at least $500 \mathrm{ml}$ and (2) creatinine $<4 \mathrm{mmol} \mathrm{dl}^{-1}$ in women or $<6 \mathrm{mmol} \mathrm{dl}^{-1}$ in men $^{46,47}$. Comparisons were conducted using the Student's $t$-test for independent samples.

Ethics. This project was registered in ClinicalTrials.gov (no. NCT01960972). The protocol and informed consent forms used in this project were reviewed and approved by the institutional review boards of Universidad Peruana Cayetano Heredia, Lima, Peru, and Johns Hopkins University, Baltimore, MD, USA. Given that the intervention was implemented at the village level, but the outcome was measured at the individual level, we involved all the members of the recruited families in the study. For this, we initially engaged with the authorities and leaders from the villages, and an initial presentation and explanation of the study at the village level were conducted before starting the research activities. Then, family members aged $\geq 18$ years were contacted for individual informed consent. As hypertension is not common among children, we did not include children and adolescents, that is any family member aged $<18$ years, in the study. Participants with a history of terminal or severe chronic kidney disease (any form of dialysis) or those taking digoxin or potassium-sparing diuretics (for heart disease), together with their families, were excluded from this study.

Reporting summary. Further information on research design is available in the Nature Research Reporting Summary linked to this article.

\section{Data availability}

Anonymized clinical and anthropometric data are available on request, subject to an internal review by J.J.M., R.H.G. and A.B.-O. to ensure that the participants' anonymity and confidentiality are protected, with completion of a data-sharing agreement, and in accordance with the Universidad Peruana Cayetano Heredia and Johns Hopkins University's institutional review boards and institutional guidelines. Material requests, that is marketing campaign information or economics data requests, will be considered based on a proposal review, and completion of a material transfer agreement and/or a data use agreement. Please submit requests for participant-related clinical and other data to A.B.-O. (Antonio.Bernabe@upch. pe), copying to J.J.M. (Jaime.Miranda@upch.pe).

\section{References}

29. Bernabe-Ortiz, A. et al. Launching a salt substitute to reduce blood pressure at the population level: a cluster randomized stepped wedge trial in Peru. Trials 15, 93 (2014).

30. Campbell, M. K., Piaggio, G., Elbourne, D. R. \& Altman, D. G. Consort 2010 statement: extension to cluster randomised trials. BMJ 345, e5661 (2012).

31. Davey, C. et al. Analysis and reporting of stepped wedge randomised controlled trials: synthesis and critical appraisal of published studies, 2010 to 2014. Trials 16, 358 (2015).
32. Hemming, K., Haines, T. P., Chilton, P. J., Girling, A. J. \& Lilford, R. J. The stepped wedge cluster randomised trial: rationale, design, analysis, and reporting. BMJ 350, h391 (2015).

33. Brown, C. A. \& Lilford, R. J. The stepped wedge trial design: a systematic review. BMC Med. Res. Methodol. 6, 54 (2006)

34. Bernabe-Ortiz, A. et al. Contribution of modifiable risk factors for hypertension and type-2 diabetes in Peruvian resource-limited settings. J. Epidemiol. Commun. Health 70, 49-55 (2016).

35. Bernabe-Ortiz, A. et al. Impact of urbanisation and altitude on the incidence of, and risk factors for, hypertension. Heart 103, 827-833 (2017).

36. Censos Nacionales 2017: XI de Población y VI de Vivienda (Instituto Nacional de Estadistica e Informatica, 2017); http://www.inei.gob.pe/estadisticas/censos

37. French J., Blair-Stevens C., Merritt R. \& McVey D. Social Marketing and Public Health: Theory and practice (Oxford Univ. Press, 2010).

38. Pretell, E. A. \& Higa, A. M. Peru celebrates 25 years of sustained elimination of IDD. IDD Newsl. 31, 12-14 (2009).

39. Coleman, A., Steel, S., Freeman, P., de Greeff, A. \& Shennan, A. Validation of the Omron M7 (HEM-780-E) oscillometric blood pressure monitoring device according to the British Hypertension Society protocol. Blood Press. Monit. 13, 49-54 (2008).

40. Chobanian, A. V. et al. The seventh report of the joint national committee on prevention, detection, evaluation, and treatment of high blood pressure: the JNC 7 report. JAMA 289, 2560-2572 (2003).

41. R Core Team. A Language and Environment for Statistical Computing (R Foundation for Statistical Computing, 2019); https://www.R-project.org

42. Hughes, J. P., Granston, T. S. \& Heagerty, P. J. Current issues in the design and analysis of stepped wedge trials. Contemp. Clin. Trials 45(Pt A), 55-60 (2015).

43. Scott, J. M., deCamp, A., Juraska, M., Fay, M. P. \& Gilbert, P. B. Finite-sample corrected generalized estimating equation of population average treatment effects in stepped wedge cluster randomized trials. Stat. Methods Med. Res. 26, 583-597 (2017).

44. Durovni, B. et al. Effect of improved tuberculosis screening and isoniazid preventive therapy on incidence of tuberculosis and death in patients with HIV in clinics in Rio de Janeiro, Brazil: a stepped wedge, cluster-randomised trial. Lancet Infect. Dis. 13, 852-858 (2013).

45. Schoenfeld, D. Partial residuals for the proportional hazards regression model. Biometrika 69, 239-241 (1982).

46. Stolarz-Skrzypek, K. et al. Fatal and nonfatal outcomes, incidence of hypertension, and blood pressure changes in relation to urinary sodium excretion. JAMA 305, 1777-1785 (2011).

47. Swanepoel, B., Schutte, A. E., Cockeran, M., Steyn, K. \& Wentzel-Viljoen, E. Sodium and potassium intake in South Africa: an evaluation of 24-hour urine collections in a white, black, and Indian population. J. Am. Soc. Hypertens. 10, 829-837 (2016).

\section{Acknowledgements}

This study was supported by the National Heart, Lung and Blood Institute (Project 5 U01 HL114180-01), under the Global Alliance for Chronic Diseases hypertension program. A.B.-O. was supported by a Wellcome Trust Research Training Fellowship in Public Health and Tropical Medicine (grant no. 103994/Z/14/Z). V.G.S.y.R. was funded by the Dirección de Gestión de la Investigación at the Pontifica Universidad Católica del Perú (grant no. DGI-2017-496)

\section{Author contributions}

A.B.-O. and J.J.M. drafted the first version of the manuscript. R.M.C.-L. provided input to this version of the manuscript. A.B.-O., R.H.G., K.A.S. and J.J.M. conceived and designed the overall study. V.G.S.y.R. and A.B.-O. developed the statistical analysis plan and conducted the statistical analysis. V.P.-L. led the social marketing campaign. M.K.C. designed the strategy for the cost-effectiveness analysis. F.D.-C. and M.A.P. conducted qualitative work during the intervention as part of a process evaluation. All the authors contributed to the revision of the manuscript for important content and gave their final approval of the version submitted for publication.

\section{Competing interests}

The authors declare no competing interests.

\section{Additional information}

Extended data is available for this paper at https://doi.org/10.1038/s41591-020-0754-2. Supplementary information is available for this paper at https://doi.org/10.1038/ s41591-020-0754-2.

Correspondence and requests for materials should be addressed to J.J.M.

Peer review information Jennifer Sargent was the primary editor on this article and managed its editorial process and peer review in collaboration with the rest of the editorial team.

Reprints and permissions information is available at www.nature.com/reprints. 

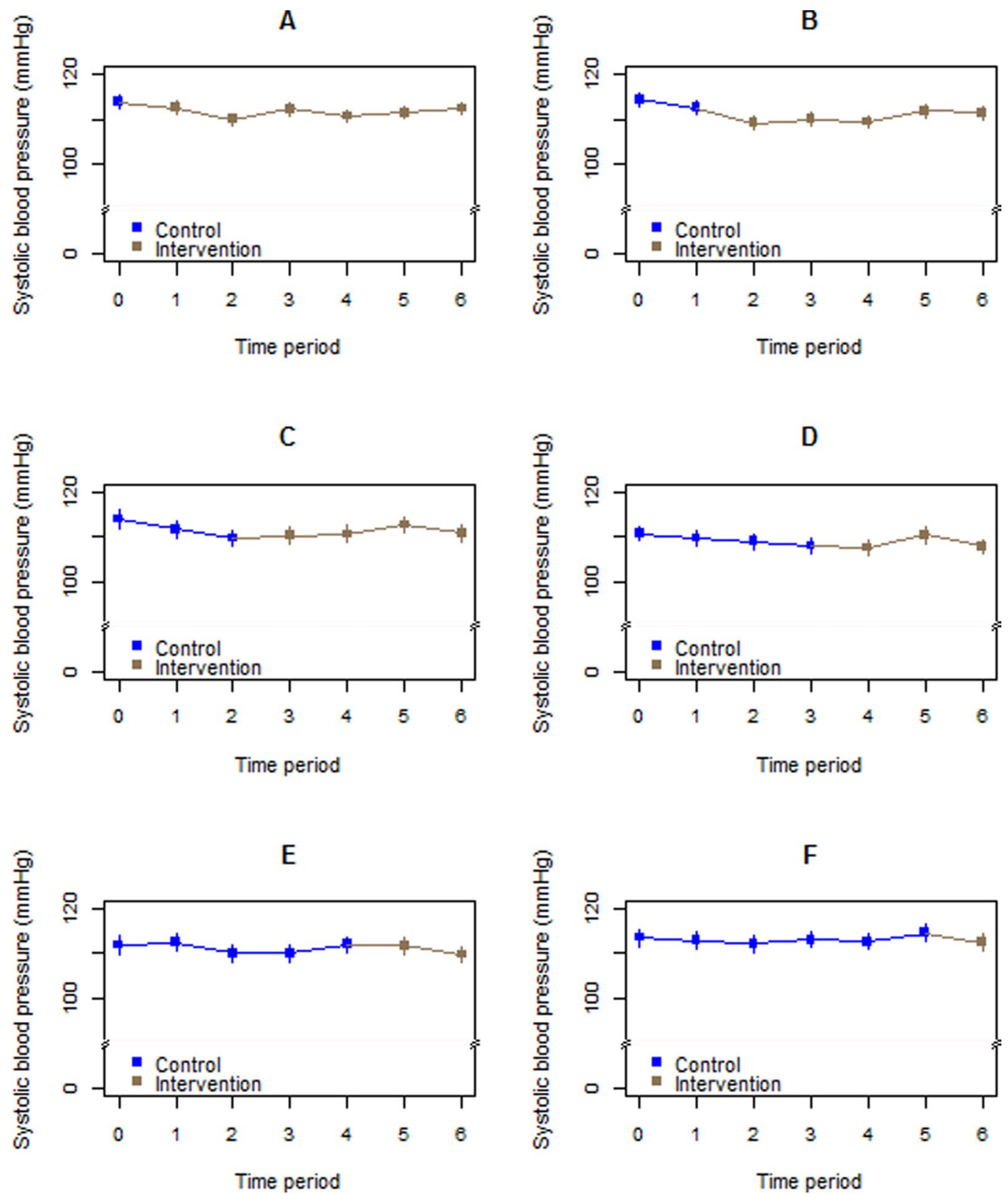

Extended Data Fig. 1 | Trends in mean SBP and their respective 95\% confidence intervals by village and intervention period. Blue symbols and lines indicate measurements and time before institution of the study intervention, and brown symbols and lines represent the intervention time periods. Time periods are 5-month analysis periods occurring before the initiation of the intervention in each wave. 



Extended Data Fig. 2 | Trends in mean DBP and their respective $\mathbf{9 5 \%}$ confidence intervals by village and intervention period. Blue symbols and lines indicate measurements and time before institution of the study intervention, and brown symbols and lines represent the intervention time periods. Time periods are 5-month analysis periods occurring before the initiation of the intervention in each wave. 


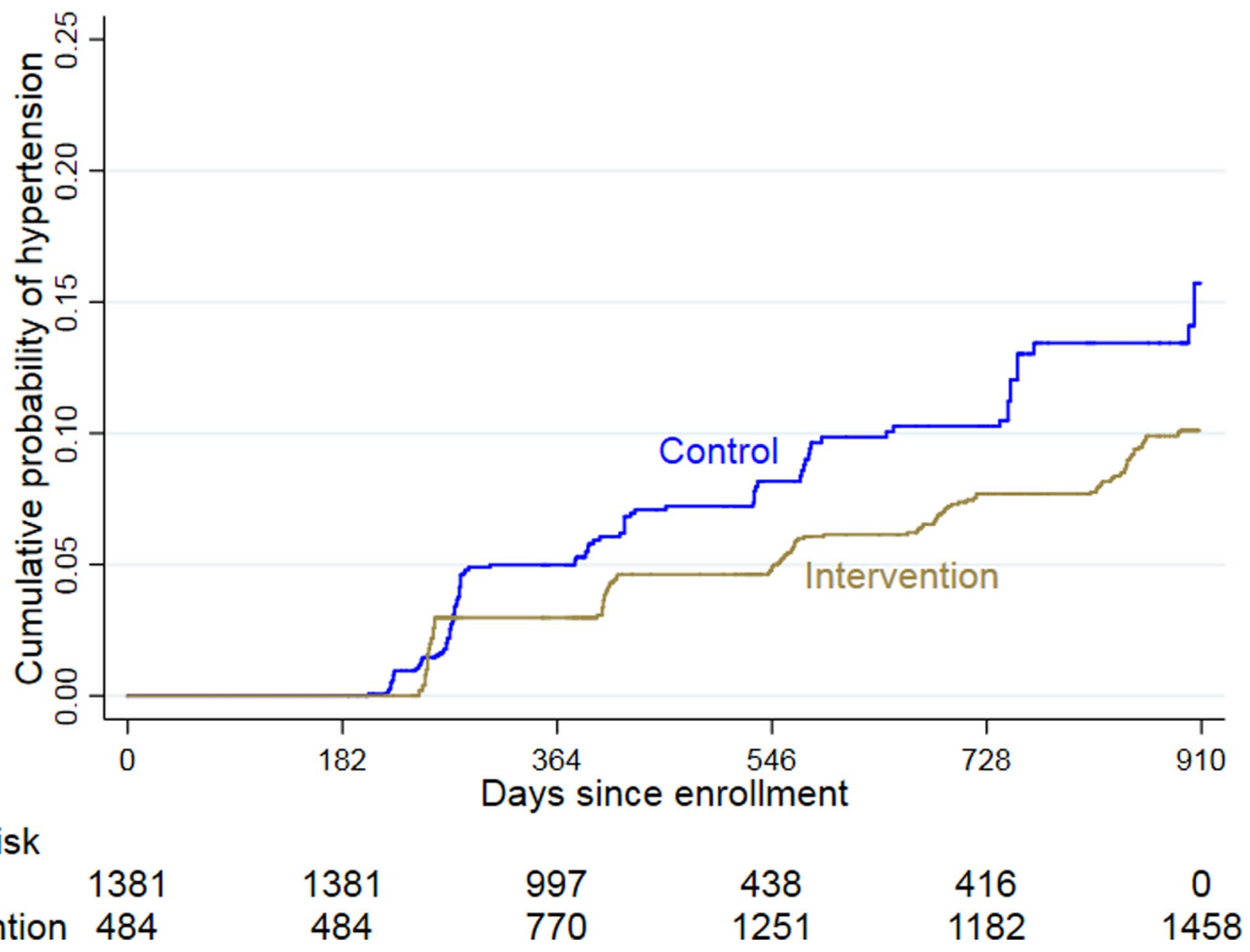

No. at risk

Control Intervention 484
484
770
1182

Extended Data Fig. 3 | Cumulative probability of developing hypertension in the intervention and control group during the duration of the study. Probability of developing hypertension in the intervention and control groups. 


\section{Reporting Summary}

Nature Research wishes to improve the reproducibility of the work that we publish. This form provides structure for consistency and transparency in reporting. For further information on Nature Research policies, see Authors \& Referees and the Editorial Policy Checklist.

\section{Statistics}

For all statistical analyses, confirm that the following items are present in the figure legend, table legend, main text, or Methods section.

$\mathrm{n} / \mathrm{a}$ Confirmed

\ The exact sample size $(n)$ for each experimental group/condition, given as a discrete number and unit of measurement

$\square$ A statement on whether measurements were taken from distinct samples or whether the same sample was measured repeatedly

$\varnothing$ The statistical test(s) used AND whether they are one- or two-sided

Only common tests should be described solely by name; describe more complex techniques in the Methods section.

$\square$ A description of all covariates tested

$\square \bigotimes$ A description of any assumptions or corrections, such as tests of normality and adjustment for multiple comparisons

$\checkmark$ A full description of the statistical parameters including central tendency (e.g. means) or other basic estimates (e.g. regression coefficient)

AND variation (e.g. standard deviation) or associated estimates of uncertainty (e.g. confidence intervals)

For null hypothesis testing, the test statistic (e.g. $F, t, r$ ) with confidence intervals, effect sizes, degrees of freedom and $P$ value noted

Give P values as exact values whenever suitable.

Х $\square$ For Bayesian analysis, information on the choice of priors and Markov chain Monte Carlo settings

Х $\square$ For hierarchical and complex designs, identification of the appropriate level for tests and full reporting of outcomes

$\triangle \square$ Estimates of effect sizes (e.g. Cohen's $d$, Pearson's $r$ ), indicating how they were calculated

Our web collection on statistics for biologists contains articles on many of the points above.

\section{Software and code}

Policy information about availability of computer code

Data collection Data was collected using paper and pen approach.

Data analysis All statistical procedures were conducted using Stata for Windows v15.0 (StataCorp, College Station, TX, US) and R statistical software.

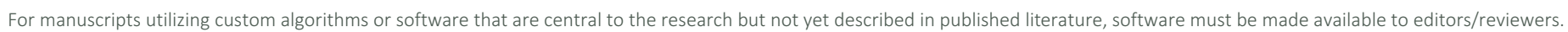
We strongly encourage code deposition in a community repository (e.g. GitHub). See the Nature Research guidelines for submitting code \& software for further information.

\section{Data}

Policy information about availability of data

All manuscripts must include a data availability statement. This statement should provide the following information, where applicable:

- Accession codes, unique identifiers, or web links for publicly available datasets

- A list of figures that have associated raw data

- A description of any restrictions on data availability

Anonymized clinical and anthropometric data are available upon request, subject to an internal review by J.J.M., R.H.G. and A.B-O. to ensure that the participants' anonymity and confidentiality are protected, completion of a data sharing agreement, and in accordance with the Universidad Peruana Cayetano Heredia and Johns Hopkins University's institutional review boards and institutional guidelines. Material requests, i.e. marketing campaign information, or economics data requests will be considered based on a proposal review, completion of a material transfer agreement and/or a data use agreement. Please submit requests for participantrelated clinical and other data to J.J.M (Jaime.Miranda@upch.pe) copying A.B-O. (Antonio.Bernabe@upch.pe). 
Please select the one below that is the best fit for your research. If you are not sure, read the appropriate sections before making your selection.

$\bigotimes$ Life sciences $\quad \square$ Behavioural \& social sciences $\quad \square$ Ecological, evolutionary \& environmental sciences

For a reference copy of the document with all sections, see nature.com/documents/nr-reporting-summary-flat.pdf

\section{Life sciences study design}

All studies must disclose on these points even when the disclosure is negative.

Sample size Calculations were derived using preliminary data from the baseline of the CRONICAS cohort study in Tumbes and the PERU MIGRANT estimates. Power for the stepped wedge design was computed for a continuous endpoint, where $\mathrm{X}$ is a N X T matrix showing the treatment pattern i.e. Xij=1 if cluster " $\mathrm{i}$ " received the intervention at time " $\mathrm{j}$ " and 0 otherwise. We assumed a significance level of $5 \%$, a standard deviation of blood pressure within sites of $20 \mathrm{mmHg}$, the number of clusters (N) of 6; the number of time periods (T) of 6 (excluding baseline assessment), the average number of subjects assessed per cluster and time period of 300 , and an approximation to the coefficient of variation of 0.20 . Based on those assumptions, we calculated a power over $90 \%$ to find a difference of $3 \mathrm{~mm} \mathrm{Hg}$ in blood pressure levels between the intervention and control groups. This magnitude of difference is within the expected range that provides major public health gains in the longterm, in particular in reduction of stroke. Typically, the coefficient of variation ranges between 0.15 and 0.40 , but when this value is unknown, as in this study, sensitivity of the sample size within this range needs to be verified. In this protocol, power calculations using both extremes of coefficient of variation yields a power greater than $90 \%$.

Data exclusions Intent to treat analysis done.

Replication Analyses were done in STATA and R to verify consistency.

Randomization The selected villages were randomly assigned to one of the six sequences (one village = one cluster) for time cross-over from control to intervention. For this, a computer-generated list of random numbers was used and information was kept in a password-protected computer. The order of villages to be implemented was revealed one by one as required according to the nature of the study.

Blinding

Due to the pragmatic nature of the intervention, the participants were not blinded; however, the primary study outcome was objectively measured using standardized techniques. A team of fieldworkers, differing from those involved with the implementation of the intervention, was responsible for periodic assessments of participants using automated devices to reduce observer bias.

\section{Reporting for specific materials, systems and methods}

We require information from authors about some types of materials, experimental systems and methods used in many studies. Here, indicate whether each material, system or method listed is relevant to your study. If you are not sure if a list item applies to your research, read the appropriate section before selecting a response.

\begin{tabular}{l|l} 
Materials \& experimental system \\
\hline n/a & Involved in the study \\
$\searrow$ & $\square$ Antibodies \\
$\searrow$ & $\square$ Eukaryotic cell lines \\
$\square$ & $\square$ Palaeontology \\
$\square$ & $\square$ Animals and other organisms \\
$\square$ & $\bigotimes$ Clinical data
\end{tabular}

\begin{tabular}{l|l} 
Methods \\
\hline n/a & Involved in the study \\
$\square$ & $\square$ ChIP-seq \\
$\square$ & $\square$ Flow cytometry \\
$\square$ & $\square$ MRI-based neuroimaging
\end{tabular}

\section{Human research participants}

\section{Policy information about studies involving human research participants}

Population characteristics Potentially eligible subjects were identified from the most updated census in the area (2010, updated in 2014). All males and females aged 18 years and over from the six selected villages, who were capable of understanding procedures, capable of providing informed consent and full-time residents in the area were eligible. Individuals with a self-reported history of chronic kidney disease and heart disease who were undergoing treatment with digoxin were excluded from the study. A total of 2376 (91.2\%) out of 2605 eligible subjects in the six villages were enrolled in the study from Apr 3 to Jul 17, 2014; 49.6\% females, mean age $43.3 \pm 17.2$ years.

Recruitment

Participant recruitment, as well as the initial assessment, was performed during the first four months of the study (April to July 2014). Individuals were contacted through home visits aiming to enroll all members of the household members of the villages who met the selection criteria. 

project were reviewed and approved by the institutional review boards of the Universidad Peruana Cayetano Heredia, Lima, Peru, and Johns Hopkins University, Baltimore, MD, USA. Given that the intervention was implemented at the village level but the outcome was measured at the individual level, we involved all the members of the recruited families in the study. For this, we initially engaged with authorities and leaders from the villages, and an initial presentation and explanation of the study at the village level was conducted before starting the research activities. Then, family members aged 18 years and over were contacted for individual informed consent. Since hypertension is not common among children, we did not include children and adolescents, i.e. any family member $<18$ years old, in the study. Participants with a history of terminal or severe chronic kidney disease (any form of dialysis) or those taking digoxin or potassium-sparing diuretics (for heart disease) with their families were excluded from this study.

Note that full information on the approval of the study protocol must also be provided in the manuscript.

\section{Clinical data}

Policy information about clinical studies

All manuscripts should comply with the ICMJE guidelines for publication of clinical research and a completed CONSORT checklist must be included with all submissions.

Clinical trial registration

Study protocol

Data collection

Outcomes

\section{NCT01960972 (clinicaltrials.gov)}

Bernabe-Ortiz A, Diez-Canseco F, Gilman RH, Cardenas MK, Sacksteder KA, Miranda JJ. Launching a salt substitute to reduce blood pressure at the population level: a cluster randomized stepped wedge trial in Peru. Trials 2014;15:93.

After providing consent, each participant was given a unique code. At baseline, detailed information regarding sociodemographics (e.g., age, sex, education, wealth index), lifestyle behaviours (smoking, alcohol consumption, and physical activity), self-reported personal medical history and medication (hypertension and type 2 diabetes mellitus), anthropometric measurements (height, weight, and blood pressure), and health-care utilization and expenditures was collected using paperbased formats. Follow-up assessments were conducted in all participants and included some lifestyle behaviours (smoking and alcohol consumption), anthropometric measurements (weight and blood pressure), and health-care utilization and expenditures. Urine samples were retrieved in a random sub-sample of 600 participants after baseline and in another randomly selected subsample of 600 participants at the end of the study. Only one participant per household was included in the urine assessments. Urine samples were collected over a 24-hour period, and all samples were assessed in a central laboratory facility. These samples were used to extract information about levels of creatinine, sodium and potassium. Sodium and potassium were assessed using the ion-selective electrode method, whereas creatinine was assessed with the compensated kinetic Jaffe method.

The primary outcomes were SBP and DBP, assessed as continuous variables (in $\mathrm{mm} \mathrm{Hg}$ ) evaluated in the period between the end of each wedge and start of the next one. Blood pressure assessments were performed with the participants seated, after a 5minute resting period, using an automated device (OMRON HEM-780, Illinois, US) that had been previously validated in adult populations.38 Three different measurements, at least one minute apart, were carried out, and the average of the second and third measurements was used for the analyses.

The secondary outcomes included progression toward hypertension (incidence) and, in a random sub-sample of participants, changes in levels of sodium and potassium excretion in 24-hour urine. Hypertension at baseline was defined as SBP $\geq 140 \mathrm{~mm} \mathrm{Hg}$, $\mathrm{DBP} \geq 90 \mathrm{~mm} \mathrm{Hg}$, a self-reported physician diagnosis or current treatment for hypertension. 39 During follow-up, hypertension was defined based in two ways: considering only the study measurements (average of the second and third measurements, with the participant in seated position, after resting five minutes, and at least one minute apart between measures), taking advantage of their repeated assessment conducted every five months, as well as using the same definition as in the baseline. 